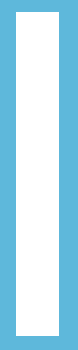

\title{
LA UNIVERSIDAD CONFESIONAL Y LOS NUEVOS MODELOS DE UNIVERSIDADES: ¿ES POSIBLE MANTENER LA IDENTIDAD?
}

\section{The Denominational University and the New University Models: ¿Is possible to keep the identity?}

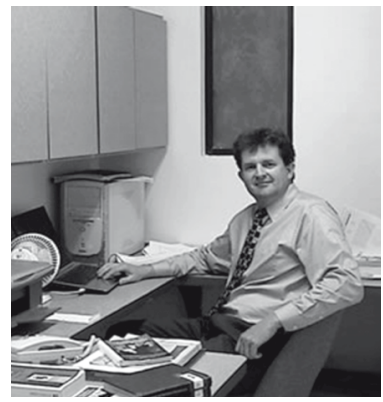

\section{Gustavo Gregorutti}

Bachiller en Ciencias Sociales por la Universidad Adventista del Plata, Ph.D. en Administración Educacional y Liderazgo por la Andrews University. Actualmente se encuentra desarrollando su segundo doctorado en la Universidad de Humboldt, Alemania; asimismo, se desempeña como docente de posgrado de la Universidad de Montemorelos, México, donde realiza actividades de investigación, produciendo publicaciones y ponencias en diversas revistas de nivel internacional. 


\section{Resumen}

Este estudio presenta la relación dinámica que se da entre las diversas concepciones misionales y su impacto sobre los modelos universitarios. El sobredimensionamiento del paradigma de producción de conocimiento para la universidad crea un desbalance educativo. La universidad confesional puede contribuir con sus características a una oferta educativa que incluya las tres misiones imperantes pero agregando, en esta interacción, el surgimiento de la cuarta misión como un aporte a una formación más completa. Este nuevo propósito misional, no solo sirve para la universidad confesional dado que incluye el fomento de la sabiduría, sino que también puede ser de utilidad a la institución laica. Hacia el final, la discusión se orienta a algunas posibles problemáticas para generar un modelo universitario más balanceado.

Palabras clave: Universidad, misión, modelo.

\section{Abstract}

This study presents the dynamic relationship between the different mission statements and their impact on university's models. The excessive promotion of the production of knowledge, as the central paradigm for higher education, has produced unbalances in universities. Religious affiliated institutions needs to reposition themself with their characteristics to offering an education that includes all three existing missions, but adds a fourth one as a way of enriching training. This new mission purpose that centers on the development of wisdom, it is not only useful for religious affiliated universities, but also for all kind of higher education models. Toward the end, the paper turns to some of the complications a more balanced model would face.

Keywords: University, mission, model. 


\section{Introducción}

Como efecto de la creciente globalización y del desarrollo de nuevos medios de comunicación, las sociedades se comportan bajo formas homogeneizantes que las Ilevan a copiar e imitar los procedimientos y prácticas que son vistas como referentes de éxito en múltiples áreas (Di Maggio y Powell, 1983). El conocimiento siempre ha sido vital para lograr un fin determinado, pero en estos últimos 30 años la generación de ideas y su impacto se ha transformado en un común denominador que toda ecuación de competencia debe incorporar. El impacto de las nuevas tecnologías ha producido una metamorfosis sobre los medios de comunicación y las interacciones humanas. La creación de riqueza está cada vez más asociada con la innovación que explica, transforma y hasta contradice los modos de administrar la realidad. Quien logra ser parte de ese majestuoso flujo de interacciones y dinámicas está más cerca de transformarse en un protagonista central en su ámbito de acción. He aquí el poder de las ideas en el siglo XXI. La actual sociedad del conocimiento sustenta como objetivo principal la generación de bienes que faciliten la vida, como queriendo hacer realidad los modelos utópicos renacentistas de un mundo idealizado casi perfecto.

La universidad del siglo XXI ha llegado a ser una pieza clave en todo este proceso de generación de ideas. Este fenómeno se ve claramente ejemplificado en el avance exponencial, en las últimas décadas, de las ciencias biomédicas y tecnológicas. Inventar una simple píldora que cura algún mal puede significar una avalancha de ingresos y prestigio para el inventor, pero también para la universidad que le facilitó los laboratorios para la invención. Las comunidades que circundan a universidades de este tipo se ven ampliamente beneficiadas por la generación de empleo con el mejoramiento de los niveles sociales. Los gobiernos recaudan más impuestos y el ciclo sigue en innumerables formas. Este podría ser la innovación más notable que las universidades hayan aportado a la sociedad. Esta es la tercera misión de la universidad, como se la denomina (Slaughter y Rhoades, 2004). Ya no basta enseñar y hacer investigación, es necesario construir puentes al mundo para modificarlo. Esta es la muerte de la Torre de Marfil (Duderstadt, 2000). La universidad como un medio transformador de la sociedad, basada en el conocimiento.

Esta tendencia global ha creado una poderosa oleada de contagio: la universidad de investigación (Research University) como modelo ejemplar para la educación superior, por sobre todas las cosas. ¿Pero qué significa una universidad que produce conocimiento transferible y comerciable? ¿Cuánto 
cuesta? ¿Es posible universalizarla? ¿Es ese el mejor modelo para todos los integrantes de la comunidad académica? ¿Qué pasa con las universidades que tienen otros objetivos misionales? Estos son algunos de los problemas que este trabajo aborda. Específicamente, el análisis se enfoca en la universidad confesional y su interacción con estas tendencias predominantes en Latinoamérica y en todo el mundo.

\section{La comercialización de la investigación: Un nuevo paradigma}

Tradicionalmente la universidad desde sus modestos inicios en Bologna, en 1088, entendida ésta como la primera institución formalmente universitaria, se ha dedicado a transmitir conocimiento más que a generarlo. Aunque se puede argumentar si en el proceso de transmisión no se producen también descubrimientos, las actividades giraban en torno a preparar gente en las artes liberales o lo que se llamaba el cuadrivium y trívium (Lucas, 1996). El conocimiento básico estaba en los libros y en unas pocas observaciones fácticas. Los alumnos y profesores debían descubrirlo y transmitirlo en una incesante cadena que duró por siglos. Este modelo de la realidad universitaria comienza a fraccionarse con la curiosidad renacentista. Uno de los mejores ejemplos de esto puede encontrarse en Leonardo Da Vinci que encarnó la incansable sed por conocer y dominar la realidad, en una época de prohibiciones. Pero no es hasta la revolución industrial europea del siglo XVIII y especialmente XIX que esta primera misión de la universidad se vio sometida a grandes cambios. Los hermanos von Humboldt en Berlín sentaron las base de una revolución que se diseminó rápidamente por todo el mundo occidental, a saber: la investigación como segunda misión académica (Veysey, 1970). La universidad no solo transmite ideas, sino que ahora las crea intencionalmente y las transmite a sus estudiantes en una interacción renovada. Los alumnos forman parte de ese proceso en torno a las cátedras que investigadores prominentes sustentan. Esto significó un importante cambio de paradigma y produjo una expansión del conocimiento nunca antes vista. La Universidad de Humboldt (Ilamada también de Berlín) produjo más de dos docenas de premios nobeles en las primeras dos décadas del siglo XX transformándose en modelo para la incipiente universidad americana. La Universidad de Chicago y la de Johns Hopkins son dos ejemplos sobresalientes que siguieron muy de cerca este enfoque. Hasta hoy en día, esta última es la que atrae más millones en aportes federales para producir investigación. ${ }^{1}$

1 Para más detalles, ver http://www.nsf.gov/statistics/infbrief/nsf10329/ 
La segunda guerra mundial atrasó poderosamente a la universidad alemana y dio cabida a la americana que ya venía desplegándose rápidamente. Uno de los motores más importantes que impulsó a la investigación americana fue la transferencia de conocimiento de las universidades a proyectos militares (Altbach, Berdahl y Gumport, 2005). El gobierno comenzó a colocar dinero para movilizar la ciencia básica (a la manera Humboldtiana) hacia la aplicación con propósitos bélicos. Poco a poco las universidades más desarrolladas en EEUU iniciaron su camino hacia la comercialización del conocimiento. Este hecho cobra notoriedad creciente sintetizándose en una serie de acciones legales que autorizan, finalmente, el lucro de la propiedad intelectual. La más renombrada fue el Acta de Bayh-Dole (1980) que formaliza lo que estaba ocurriendo en la práctica. Así, la universidad americana puede, legalmente, comercializar su producción intelectual, aun cuando se haya usado fondos federales. Este simple hecho ha tenido implicancias que van más allá de la universidad americana. El nuevo modelo promovía la tercera misión de la universidad, a saber la comercialización y transferencia de ideas a la sociedad para transformarla, especialmente en lo económico. Este prototipo ha probado ser altamente efectivo para un puñado de instituciones con abundante recursos financieros y humanos (Slaugher y Rhoades, 2004). Así, la industria, el gobierno y la universidad se unen en una nueva aventura para crear conocimiento comercializable y producir nuevos empleos. Etzkowitz (1996) Ilamó esta interacción la "Triple Hélice" que representa una trilogía de actores.

Con la llegada y afianzamiento de la globalización, el intercambio favorecido por la tecnología impulsa la universalización de modelos exitosos. Las economías emergentes buscan modos de competir y mejorar sus economías en un mercado cada vez más global. ¿Cómo hacer o adónde ir para ser más competitivos? La respuesta está en el patio de atrás de cada gobierno: la universidad. Pero no cualquier institución, claro está. Son universidades que enfatizan fuertemente la producción de ideas y se orientan esencialmente a este propósito con los mejores docentes, alumnos y recursos. Las políticas neoliberales promovidas por grandes políticos como Margaret Thatcher y Ronald Reagan, en los 80s acompañadas por los grandes sistemas internacionales de financiamiento como el Banco Mundial, el FMI y otros (Toakley, 2004) aceleraron la difusión de este modelo. En realidad, ha cobrado una preeminencia tal que se ha casi elevado al nivel de creencia religiosa con características dogmáticas. Prueba de ello son los rankings internacionales, léase The Ti- 
mes, QS, Shanghái, entre otros, que dan un peso preponderante a la producción y transferencia de conocimiento para jerarquizar la calidad de una institución.

Para entender mejor estas dinámicas, el gráfico 1 explica la lógica que las universidades parecen estar siguiendo. Las abscisas representan el paradigma de los productores versus los consumidores de conocimiento. Las ordenadas miden el énfasis o implicancia económica versus un enfoque más humanista de los modelos universitarios. Estas dos coordenadas están representando funciones misionales, como un continuo con múltiples posibilidades que generan diversos modelos. Cada uno de los cuadrantes combina uno de los dos parámetros guía; de izquierda a derecha (predominante) y de abajo hacia arriba (predominante). En la medida que las misiones institucionales se mueven hacia arriba y hacia la derecha, las universidades se clasifican como más prestigiosas (rankings) y como más relevantes económicamente en el contexto de una universidad orientada a la producción de ideas. Los rankings y las clasificaciones de la calidad son directamente proporcional a la orientación hacia la tercera misión. Por el contrario, las instituciones que están mayormente sobre la izquierda de la abscisa enfatizan más la formación de recursos humanos con una fuerte transmisión de conocimiento como paradigma imperante. De la misma manera, se puede ver la orientación, en la ordenada, hacia un mayor o menor grado de impacto económico en la preparación de recursos humanos expresado en una misión más o menos humanística.

Gráfico 1. Dimensiones misionales de las universidades del siglo XXI

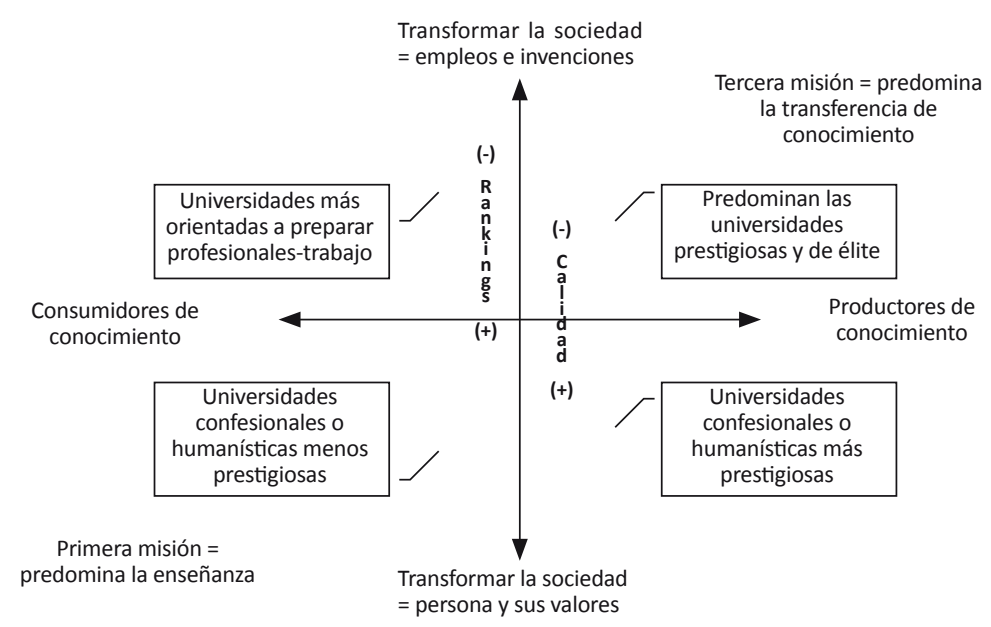


Las instituciones se posicionan, como ya se mencionó, en los cuadrantes dependiendo de las combinaciones misionales que las orientan. Las universidades confesionales, por su constitución misional, tienden a estar en los cuadrantes inferiores aunque en algunos casos, algunas de ellas se han secularizado tanto que no se distinguen mucho de las más prestigiosas. El cuadro sugiere que en la medida que una universidad se enfoca a la enseñanza y adhiere al paradigma humanístico-religioso, será percibida y clasificada como de calidad inferior.

\section{Necesidad de modelos alternativos: La universidad confe- sional}

Pero, ¿pueden todas las universidades buscar el modelo más prestigioso? O mejor aún, ¿es deseable este modelo para todas las instituciones de educación superior? Ahora, ¿qué sucede con las otras instituciones que no han llegado a la tercera misión? El isomorfismo institucional está produciendo una fuerte presión sobre las instituciones que no representan este modelo y las categoriza como de calidad inferior (Gregorutti, 2010b). Esto tiene importantes implicaciones prácticas. La solución a estas preguntas se puede hallar en la coexistencia de modelos paralelos para suplir las múltiples demandas de la sociedad, como lo representa el gráfico 1 . Entonces, la universidad confesional debería posicionarse como una alternativa válida. Si esto es posible, ¿cuáles serían la características distintivas de una universidad confesional del siglo XXI? Esta pregunta se la puede aproximar desde los siguientes aspectos, a saber:

1. Cosmovisión. La universidad confesional contempla la tarea educativa no solamente como la búsqueda de un entrenamiento para conseguir un trabajo y ser activos económicamente. La epistemología de este tipo de instituciones no rechaza lo espiritual porque no es medible, más bien lo incorpora para dar a los estudiantes todas las dimensiones de la realidad con una base de valores espirituales y profesionales.

2. Propósito. Busca el desarrollo del carácter del estudiante basado en una experiencia religiosa con Dios. En ese proceso, se producen cambios de valores en los jóvenes que tendrán impacto en las prácticas laborales posteriores. La naturaleza humana necesita de la intervención divina para corregir el carácter. Así, el entrenamiento profesional y científico se desarrolla integrándolos con la construcción de valores. El propósito de estas instituciones se lo puede igualar a una cosmovisión renovada que se expresa e infunde a todos sus actores.

3. Currículo. Incorpora literaturas y conocimientos largamente dejados de lado 
por las corrientes positivistas de las ciencias modernas. Se estudian libros sagrados como fuente de sabiduría para la vida. Se basa en una integración de ciencia y fe para el desarrollo de valores que forman a la persona y no solamente a un profesional.

4. Los docentes. Son una combinación de profesionales con valores y vidas ejemplares que sientan modelos para los jóvenes que buscan un mejor porvenir. Al mismo tiempo, estos profesores son referentes profesionales para la comunidad científica y académica en la que se desempeñan.

5. Los alumnos. Reconocen que la dimensión espiritual está presente en sus vidas y desean desarrollarla en interacción con la disciplina que estudian, los docentes, amigos y experiencias co-curriculares que la universidad brinda.

6. Interacción con la cultura. La universidad se posiciona como referente regional, nacional o aun internacional para promover una visión proactiva del paradigma que sustenta. Al producir cambios positivos en los estudiantes, se transforma, entonces, en una agencia de renovación científica y social. Es en última instancia un difusora de una contracultura académica con impacto en todas las dimensiones del quehacer humano.

Estos elementos conforman la estructura básica para que una institución terciaria confesional se desarrolle con una propuesta alternativa a los otros modelos y genere cambios en la sociedad (Holmes, 2000). Claro está que no todas las universidades confesionales se constituyen de todos estos aspectos. Muchas van dejando sus postulados misionales básicos de lado y se alinean con lo que se promueve abiertamente como "el modelo" imperante de universidad (Benne, 2001).

Como se ha visto hasta aquí, las universidades han tenido un devenir que se inició con la transmisión de ideas, luego la producción de conocimiento para últimamente la comercialización de la producción intelectual para producir impacto social. La universidad confesional debería, hasta cierto punto, participar de estos ideales porque en sí son positivos. Sin embargo, la "genética" misional de la misma agrega una dimensión que puede ser llamada de "sabiduría". Esta componente es evitada por la gran mayoría de los sistema educativos existentes. El conocimiento por sí mismo no es suficiente; se necesita de una persona íntegra y balanceada con altas dosis de "sabiduría" para que la información no sea malversada y tenga efectos contraproducentes. Por ejemplo, muchas de las grandes estafas y procesos de corrupción fueron realizados por personas muy entrenadas y capaces, pero que no tenían escrúpulos claros. La crisis que sufrió EEUU en el 2008 estuvo muy relacionada con la erosión moral de los 
grandes CEO de los poderosos grupos económicos americanos.

El descubrimiento de la dinamita, por Alfred Nobel fue un paso hacia delante para la química industrial, pero en manos de los bandidos fue un medio de destrucción y muerte. Lo mismo se puede decir de la radioactividad controlada y su inevitable uso bélico. La creación de conocimiento sin parámetros de referencia morales no sirve de mucho. Apoyando esta idea, aunque no asociado a una iglesia en particular, Kronman (2007) argumenta que la gran mayoría de las universidades en EEUU han perdido la dimensión de "sentido de vida". Es decir, que se han transformado en escuelas de entrenamiento profesional sin tener en cuenta las otras dimensiones, como por ejemplo la espiritual y de valores para la vida. De acuerdo a Yang (2003), esta tendencia se da también en todo el mundo. El gráfico 2 representa, sobre el mismo modelo de dimensiones propuesto más arriba, lo que podría ser la cuarta misión. Las flechas azules le dan profundidad a cada uno de los modelos misionales. Esta dimensión tridimensional permite múltiples modelos de universidades que se orientarían en alguno de los cuatro cuadrantes, pero que buscan instilar sabiduría en los estudiantes. Específicamente, esta idea de buscar el desarrollo de la "sabiduría" en los alumnos o conocimiento para la vida constituye una "nueva" o cuarta misión que le daría a la universidad la oportunidad de una formación integral que retoma la importancia de los valores en la educación superior.

Gráfico 2. La cuarta misión de la universidad del siglo XXI

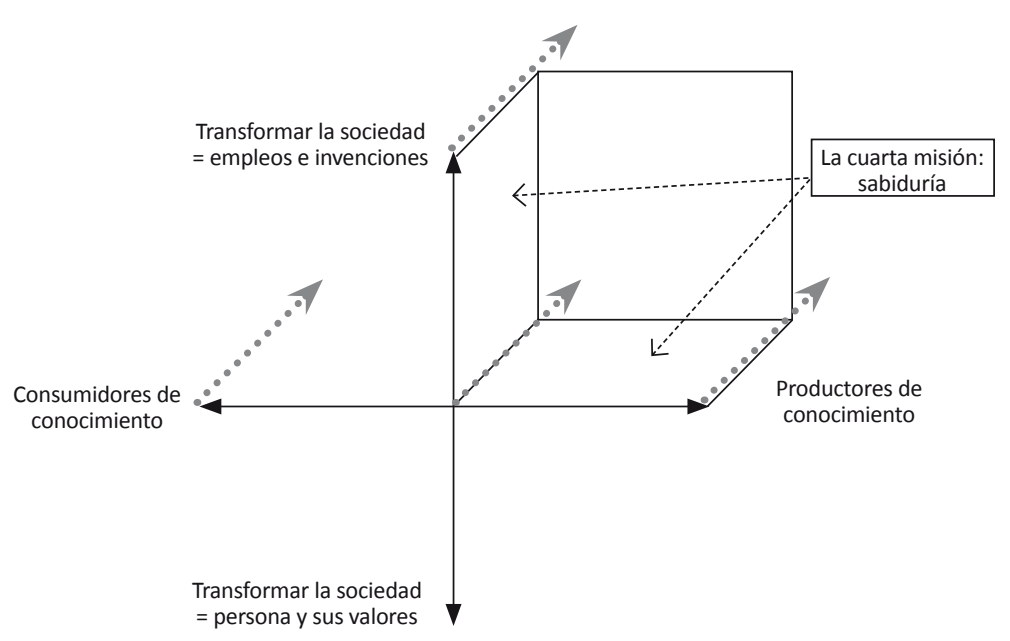


La componente de sabiduría es probablemente una de las contribuciones misionales esenciales de la universidad confesional; pero no debería ser circunscripto a este tipo de instituciones solamente. Desde un punto de vista laico, las universidades públicas y privadas en general podrían introducir aspectos globales de lo que constituye la construcción de la "sabiduría" entre los alumnos. Ejemplo de ello lo pueden plasmar la ecología, el uso de los recursos económicos con sensibilidad social y la creación de organizaciones para educar y orientar a los menos favorecidos. Todo esto por contraposición al capitalismo salvaje que muchas compañías practican. Definidamente esta dimensión misional puede ser un plus invalorable para la universidad del siglo XIX.

\section{Desafíos e ideas finales}

Las instituciones confesionales tienden a estar ubicadas en el cuadrante inferior izquierdo del gráfico 1. La actividad preponderante está centrada en la enseñanza y la formación de profesionales. Algunas universidades se han desplazado hacia la derecha incorporando la segunda misión y algunas de ellas han adoptado la tercera misión también. Es de esperar que la cuarta misión no sea un problema para la mayor parte de las instituciones de este tipo. Sin embargo, parecen tener problemas con la segunda y especialmente la tercera (Gregorutti, 2010). Agregar una nueva misión a las tres existentes puede crear muchas resistencias. Para entender las "mutaciones" de la "genética" interna de una institución terciaria, la metáfora de la "compuerta" de un dique puede ser adecuada para explicar el paradigma de cambio y evolución institucionales. De acuerdo a Schriewer (2003), para entender cómo las instituciones reaccionan a las transformaciones producidas en un mundo globalizado, se dan, lo que él llama, las "externalizaciones" de lo que acontece alrededor. Es decir que cuando las universidades buscan nuevos significados o formas de sobrevivencia, nunca lo hacen copiando totalmente un determinado modo alternativo. Como la "compuerta" de un dique "filtran" los contenidos y alternativas de acuerdo a las tradiciones e ideas internas para regular el flujo del cambio para no ser "inundados" con innovaciones foráneas. En otras palabras, las universidades pueden "mutar" dependiendo de las necesidades internas y contextuales. Este filtrado de flujos que las universidades efectúan hace extremadamente difícil definir con exactitud el momento de transición de una organización educativa. Para evitar el caos ideológico y administracional se debe buscar una reformulación colegiada y consensuada de lo que significaría agregar una 
nueva misión a las existentes. Todos sus actores deben ver la "quinta esencia" y hacerla suya a través de una planificación que plasme la misión en todas sus dimensiones institucionales. Es imperativo hacer evidente las ideas y no dejarlas a la deriva. Si no se hace esto, es probable que se divague entre alternativas contradictorias tanto en lo personal como en lo institucional.

Por otra parte, la universidad orientada a la investigación debe balancear su propuesta con, por ejemplo, la cuarta misión y entender que la producción y comercialización de ideas no es el modelo a seguir para todas las instituciones. Diversos estándares de calidad y ranking deberían ser producidos para medir las instituciones según la orientación misional preponderante. No se puede medir a todos con la misma vara, para hacer justicia a la pluralidad de instituciones. Esto último tiene una gran trascendencia para las universidades que se insertan en un mundo muy competitivo, evitando distorsiones de lo que es más relevante y prioritario. La calidad es un concepto polisémico y está muy lejos de ser objetivo, como algunos rankings dejan entrever. Esto es especialmente cierto para las instituciones que buscan acreditaciones oficiales. La presión por "acomodarse" es muy grande y puede ser distorsionar los paradigmas esenciales que las constituyen (Gregorutti, 2011). La diversidad de modelos desarrollados desde diversas propuestas misionales es altamente deseable para suplir todas las dimensiones de la persona y de la sociedad. Tal vez, el mayor desafío que enfrentan las instituciones confesionales sea justamente el de "alinearse" a las tendencias imperantes para ser relevantes sin poner en juego sus características fundamentales. 


\section{Referencias}

Altbach, P., Berdahl, R. y Gumport, P. (2005). American higher education in the twenty-first century ( $2^{\text {nd }}$ ed) (Coord). Baltimore, MD: The Johns Hopkins University Press.

Bayh-Dole Act, 35 U.S.C. § 200-212 (1980).

Benne, R. (2001). Quality with soul: how six premier colleges and universities keep faith with their religious traditions. Grand Rapids, MI: Eederman Publishing.

Di Maggio, P. y Powell, W. (1983). The iron cage revisited: Institutional isomorphism and collective rationality in organizational fields. American Sociological Review, 48, 147-160.

Duderstadt, J. (2000). A university for the $21^{\text {st }}$ century. Ann Arbor, MI: University of Michigan Press.

Etzkowitz, H. (1996). From knowledge flows to the triple helix. The transformation of academicindustrial relations in the USA. Industry \& Higher Education, 10(6), 337-342.

Gregorutti, G. (2010a). Moving from a predominantly teaching oriented culture to a research Productivity mission: The case of Mexico and the United States. Excellence in Higher Education, 1, 69-83.

Gregorutti, G. (2010b). La acreditación de los posgrados en instituciones privadas de educación superior mexicanas. Reencuentro, 59, 62-69.

Gregorutti, G. (2011). Following the path: From teaching to research University. Newcastle: Cambridge Scholars Publishing.

Holmes, A. (2000). The idea of Christian college. Grand Rapids: Eederman Publishing.

Kronman, A. (2007). Education's end: Why our colleges and universities have given up on the meaning of life. New Haven, CT: Yale University Press.

Lucas, C. (1996). American higher education: A history. New York, NY: St. Martin's Griffin.

Schriewer, J. (2003). Globalization in education: Process and discourse. Policy Futures in Education, 1(2), 271-283.

Slaughter, S. y Rhoades, G. (2004). Academic capitalism and the new economy: Markets, state, and higher education. Baltimore: Johns Hopkins University Press.

Toakley, A. R. (2004). Globalization, sustainable development and universities. Higher Education Policy, 17, 311-324.

Veysey, L. (1970). The emergence of the American university. Chicago, IL: The University of Chicago Press.

Yang, R. (2003). Globalization and higher education development: A critical analysis. International Review of Education, 49(3-4), 269-291. 